\title{
Limiar anaeróbio de jogadores de futebol de diferentes categorias
}

\author{
Anaerobic threshold in different categories \\ of soccer players
}

\author{
Daniel Barbosa Coelho \\ Lucas de Ávila Carvalho Fleury Mortimer \\ Luciano Antonacci Condessa \\ Danusa Dias Soares \\ Cristiano Lino Monteiro de Barros 1,2 \\ Emerson Silami Garcia ${ }^{1}$
}

1 Universidade Federal de Minas Gerais. Escola de Educação Física, Fisioterapia e Terapia Ocupacional. Laboratório de Fisiologia do Exercício. Belo Horizonte, MG. Brasil.

2 Centro Universitário de Patos de Minas. Patos de Minas, MG. Brasil.

Recebido em 22/01/08

Aprovado em 10/07/08
Resumo - Diferentes variáveis podem ser utilizadas para a identificação e monitoramento da capacidade aeróbia em esportes coletivos, sendo o limiar anaeróbio (LAN) muito utilizado em atividades como futebol, basquete, handebol entre outros. O objetivo do estudo foi determinar a intensidade do LAN de jogadores de futebol de diferentes categorias, avaliando os parâmetros velocidade, freqüência cardíaca (FC) e percentual da FC máxima (\%FCmáx), bem como comparar estas intensidades entre as categorias. Participaram do estudo 19 jogadores da categoria sub-17, 12 jogadores Sub-20 e 14 profissionais. O LAN foi determinado a partir da intensidade de $4 \mathrm{mM}$ obtida em um teste de campo. Este consistiu em corridas de $1000 \mathrm{~m}$, em velocidades constantes a cada tentativa. A FC e lactato ([Lactato]) obtidos no teste foram analisados através de interpolação linear. Foram determinados a velocidade, a FC e \%FCmáx correspondentes à intensidade do LAN. Identificou-se que a FCmáx dos jogadores Sub-17 (202 $\pm 7 \mathrm{bpm})$ foi maior quando comparada à categoria Profissional $(193 \pm 10 \mathrm{bpm})(\mathrm{p}<0,05)$. O LAN expresso em \%FCmáx dos jogadores Sub-17 $(87,0 \pm 1,1)$ e Sub-20 $(86,3 \pm 0,8)$ foi menor quando comparado à categoria Profissional $(91,2 \pm 1,1)(p<0,05)$. A intensidade média do LAN foi $87 \%$ FCmáx. Não foram identificadas diferenças entre as categorias quando o LAN foi considerado em bpm e como velocidade de corrida. Concluiu-se que a intensidade do LAN neste grupo pode ser considerada alta, sendo que os jogadores profissionais apresentam LAN mais alto em comparação com as categorias Sub-17 e Sub-20.

Palavras-chave: Limiar anaeróbio; Futebol; Freqüência cardíaca.

Abstract - Different variables can be used for the identification and monitoring of aerobic capacity in collective sports, with the anaerobic threshold (AT) being commonly used in long-duration team sports such as soccer, basketball, and handball. The aim of this study was to determine AT intensity in different categories of soccer players by evaluating running speed, heart rate (HR) and percent maximum HR (\%HRmax), and to compare these intensities between three soccer categories. Nineteen U-17, 12 U-20 and 14 professional players volunteered for this study. The AT was defined as the exercise intensity at which blood lactate reaches a concentration of $4 \mathrm{mM}$ (OBLA). AT was determined in a field test consisting of two to five 1000-m runs at constant speed. HR and blood lactate concentration recorded during the test were analyzed by linear interpolation. The running speed, HR and \%HRmax at OBLA were determined. HRmax was higher in U-17 players $(202 \pm 7$ bpm) than in professional players (193 \pm $10 \mathrm{bpm})(p<0.05)$. AT expressed as \%HRmax was lower in the U-17 $(87.0 \pm 1.1)$ and $U-20(86.3 \pm 0.8)$ categories when compared to the professional category $(91.2 \pm 1.1)$ $(p<0.05)$. Mean AT was $87 \%$ HRmax. No differences between categories were observed when AT was expressed as absolute HR values (bpm) or running speed. The intensity of AT is considered to be high in this group, with professional players presenting a higher AT than U-17 and U-20 players.

Key words: Anaerobic threshold; Soccer; Heart rate. 


\section{INTRODUÇÃO}

O futebol é considerado como uma atividade intermitente de alta intensidade ${ }^{1}$ e é caracterizado por corridas em alta velocidade e curta duração, saltos, cabeceios e disputas de bola, sendo que todas estas atividades demandam um alto grau de desenvolvimento de força e potência muscular. No entanto, durante mais da metade de um jogo de futebol, os jogadores estão parados ou andando. Por estas características e pela longa duração do jogo, este esporte exige dos atletas, além de um bom nível técnico, um bom condicionamento físico ${ }^{2}$.

No futebol, a demanda energética é alta se consideradas a intensidade e duração da atividade e esta pode ser avaliada pela mensuração da frequiência cardíaca (FC) em jogos ${ }^{1}$. O futebol profissional também é caracterizado por grande exigência do metabolismo anaeróbio, que pode ser evidenciada pelas altas concentrações de lactato durante um jogo $(10 \mathrm{a} 12 \mathrm{mM})$ durante o primeiro e segundo tempo, respectivamente ${ }^{3}$.

Pela intensidade e longa duração de um jogo de futebol, os jogadores devem ser capazes de manter um alto índice de esforço ao longo do mesmo. No entanto, identifica-se um declínio na distância percorrida, na intensidade de trabalho, na FC e nas concentrações de glicogênio muscular no decorrer da atividade ${ }^{2}$, sendo estas variáveis utilizadas para a determinação da demanda fisiológica neste esporte. Em decorrência destas observações, os jogadores, conseqüentemente, permanecem um menor percentual do tempo de jogo em zonas de maior intensidade de esforço no decorrer do mesmo 4 .

Wisloff et al. ${ }^{2}$ argumentam que pela duração e grande exigência física de um jogo de futebol, seria inviável que os jogadores se esforçassem tanto tempo em intensidades tão altas, pelo fato de provavelmente os atletas estarem se exercitando em uma intensidade muito próxima ou acima do limiar anaeróbio (LAN).

Uma grande capacidade aeróbica, representada através de um elevado consumo máximo de oxigênio $\left(\mathrm{VO}_{2 \operatorname{máx}}\right)$, indica que os jogadores poderiam manter um alto nível de esforço ao longo de um jogo de futebol ${ }^{5}$. Entretanto, desde 1984, o desempenho em esportes com predominância aeróbica não tem sido considerado dependente apenas do $\mathrm{VO}_{2 \max }{ }^{6}$. Segundo estes autores, o VO$2 \max$, o limiar anaeróbio e a economia de corrida, em conjunto, é que vão determinar o desempe- nho do indivíduo. Desta forma, no futebol, o desempenho aeróbio do jogador parece também depender destes três parâmetros $\left(\mathrm{VO}_{2 \max }\right.$, limiar anaeróbio e economia de corrida).

O LAN é considerado como a intensidade de exercício anterior àquela na qual se verifica um aumento exponencial da ([Lactato]) em função do aumento linear da intensidade de esforço. $\mathrm{O}$ aumento na [Lactato] acarreta em aumento na concentração de íons hidrogênio com conseqüente redução no pH. Além disso, acima da intensidade do LAN há um aumento exponencial na ventilação, na produção de gás carbônico e na concentração de catecolaminas. Todos estes fatores podem antecipar a instalação da fadiga, comprometendo assim o rendimento do atleta? Q Quanto maior a intensidade do exercício realizado acima do LAN, mais rapidamente ocorre a instalação da fadiga decorrente da acidose metabólica.

Comumente, o LAN está associado a diferentes variáveis, como velocidade de corrida, potência, freqüência ventilatória, $\mathrm{FC}, \mathrm{VO}_{2 \text { máx }}$ ou [Lactato] ${ }^{8}$.

Destas variáveis, a velocidade de corrida correspondente a [Lactato] de $4 \mathrm{mM}$ tem sido considerada como um bom parâmetro para o LAN. Sjodin e Jacobs ${ }^{9}$ denominaram a intensidade referente à [Lactato] de $4 \mathrm{mM}$ como OBLA (onset of blood lactate accumulation). Heck et al. ${ }^{10}$ verificaram que a média da lactacidemia na máxima fase estável do lactato (MFEL) dos atletas foi $4 \mathrm{mM}$, o que corresponde ao máximo balanço entre a produção e a remoção de lactato. No entanto, no estudo de Heck et al..$^{10}$ houve variação individual na lactacidemia de 3,0 a 5,5 mM na intensidade referente à MFEL. Denadai et al. ${ }^{11}$ não encontraram diferenças entre a [Lactato] de 3,5mM e a MFEL em jogadores de futebol.

É comum no futebol que a determinação do OBLA seja relacionada a uma dada velocidade ou FC nesta intensidade. A determinação destes fatores desempenha uma importante função no acompanhamento do desenvolvimento da capacidade aeróbica de jogadores ${ }^{12,13}$. Quanto maior o condicionamento aeróbico do atleta, maiores intensidades de exercício podem ser toleradas antes que o LAN seja alcançado ou ultrapassado. Desta forma, o LAN pode ser visto como um parâmetro de condicionamento aeróbico e, sua determinação deve levar em consideração a especificidade do exercício realizado? 
Das várias formas de identificação do LAN em jogadores utilizadas até então, têm-se encontrado valores em torno de $80 \%$ do $\mathrm{VO}_{2 \text { máx }}{ }^{13-15}, 170$ bpm ou $90 \% \mathrm{FC}_{\text {máx }}{ }^{14,16}$, ou $14 \mathrm{~km} / \mathrm{h}^{13}$, apresentando diferenças dependendo da função tática desempenhada pelos jogadores em campo ${ }^{17}$. Considerando que a determinação do LAN é um importante fator para o planejamento e monitoramento do treinamento físico no futebol de campo e devido à escassez de estudos desta natureza com jogadores e equipes do futebol brasileiro, os objetivos do presente estudo foram determinar a intensidade do LAN das categorias Sub-17, Sub-20 e Profissional, utilizando os

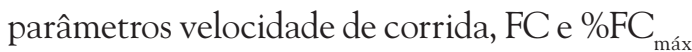
e, posteriormente, comparar estas intensidades entre as três categorias avaliadas.

Deve-se considerar que apesar do LAN identificado através do OBLA ser um parâmetro fisiológico que tem sido muito utilizado enquanto indicador de desempenho aeróbico de jogadores e como fator de controle da intensidade de treinamento, o mesmo apresenta fatores limitantes como a variação individual na [Lactato] correspondente à MFEL.

\section{PROCEDIMENTOS METODOLÓGICOS}

Este estudo foi aprovado pelo Comitê de Ética em Pesquisa (COEP) da Universidade Federal de Minas Gerais (ETIC-476/2004) e respeitou todas as normas estabelecidas pelo Conselho Nacional da Saúde (Res. 196/96), envolvendo pesquisas com seres humanos. Foi obtido de cada voluntário, após esclarecimento de todas as dúvidas provenientes da leitura do mesmo, um consentimento livre e esclarecido por escrito para a participação no experimento.

\section{Amostra}

Participaram do estudo, jogadores de futebol vinculados a uma equipe da primeira divisão do futebol brasileiro inscrita na Confederação Brasileira de Futebol (C.B.F.) (Tabela 1). Os jogadores de futebol treinavam em média 16 horas/semana e possuíam em média 5,5 \pm 4,0 anos de experiência com futebol, e grande parte dos jogadores já fazia parte dos times de categoria de base do clube em questão.

\section{Procedimentos}

$\mathrm{A} \mathrm{FC}_{\text {máx }}$ foi determinada como a maior FC encontrada dentre as três situações descritas abaixo:
1) Teste de esforço em corrida com velocidade subjetiva máxima: realizado em terreno gramado plano com o uso de chuteiras, em uma distância de 1000 metros $^{18}$.

2) Teste para a estimativa do consumo máximo oxigênio $\left(\mathrm{VO}_{2 \text { máx }}\right)^{19}$ : consistiu em percorrer uma distância de 2400 metros em terreno plano no menor tempo possível.

3) $\mathrm{FC}_{\text {máx }}$ durante jogos de uma competição oficial: a maior FC de cada jogador registrada ao longo dos jogos avaliados durante uma competição oficial (29 jogos) na qual o time ao qual os jogadores estavam vinculados participou na temporada do estudo, foi considerada como a maior $\mathrm{FC}_{\text {máx }}$ durante os jogos. Cada jogador participou em média de $4 \pm 2$ jogos.

O LAN foi determinado a partir da intensidade do OBLA obtida em um teste de campo ${ }^{20}$. O teste consistiu em duas a cinco corridas de $1000 \mathrm{~m}$ com velocidades constantes. As velocidades eram aumentadas progressivamente em $1 \mathrm{~km} / \mathrm{h}$. O percurso em que as corridas foram realizadas foi demarcado em terreno gramado, em formato circular, com marcações a cada $50 \mathrm{~m}$. O avaliador controlava a velocidade do atleta a cada $50 \mathrm{~m}$ através de estímulos verbais. Entre 60 e 90 segundos após o término de cada corrida, uma amostra de sangue era coletada ( 25 $\mu \mathrm{L})$ e a [Lactato] analisada através de um lactímetro portátil $\left(\right.$ Accusport $\left.^{\circledR}\right)$. Caso a [Lactato] alcançasse ou superasse o valor de $4 \mathrm{mM}$, o teste era interrompido. Caso contrário, o voluntário realizava mais uma corrida com um incremento de $1 \mathrm{~km} / \mathrm{h}$ na velocidade. Para garantir que a lactacidemia não ultrapassasse $4 \mathrm{mM}$, na primeira corrida, o teste iniciou a $10 \mathrm{~km} / \mathrm{h}$.

A FC foi monitorada durante todo o teste através de um cardiofrequencímetro (Polar Electro $\mathrm{Oy}^{\circledR}$, Vantage, Finland). Os valores de FC e [Lactato], registrados durante o teste foram posteriormente analisados, utilizando-se o software Microsoft ${ }^{\circledR}$ Excel 2000 para a realização da interpolação linear e determinação do OBLA.

A FC correspondente ao OBLA foi utilizada para identificar o esforço dos jogadores no LAN tanto em valores absolutos (bpm) quanto em percentual da $\mathrm{FC}_{\text {máx }}\left(\% \mathrm{FC}_{\text {máx }}\right)$. Recomenda-se que a comparação entre a intensidade de esforço dos indivíduos e dos grupos deve ser feita através dos valores relativos de $\% \mathrm{FC}_{\text {máx }}{ }^{21}$. 
O consumo máximo de oxigênio dos atletas foi estimado através de uma corrida em uma distância pré-determinada no menor tempo possível $^{19}$. Utilizou-se, no presente estudo, a distância de 2400 metros.

A coleta de dados foi realizada durante o início da temporada de treinamento das equipes das quais os jogadores faziam parte.

\section{Análise estatística}

Para a comparação dos valores de velocidade, $\mathrm{FC}, \% \mathrm{FC}_{\text {máx }}$ médio, correspondentes ao OBLA, bem como dos valores de $\mathrm{FC}_{\text {máx }}$, foi aplicada uma Análise de Variância de um fator (oneway), seguida do teste de post-hoc de Tukey, quando apropriado. Os parâmetros de caracterização da amostra, bem como os outros valores, são apresentados em média e desvio padrão. $\mathrm{O}$ nível de significância adotado foi p<0,05.

\section{RESULTADOS}

Os resultados do presente estudo estão apresentados na Tabela 2. A FC máx $_{\text {dos jogadores da }}$ categoria Sub-17 foi maior quando comparada à categoria Profissional $(p<0,05)$. O LAN expresso em \%FC ${ }_{\text {máx }}$ dos jogadores das categorias Sub-17 e Sub-20 foi menor quando comparada à categoria Profissional $(p<0,05)$. No entanto, quando este foi apresentado como valores absolutos de FC, não foram encontradas diferenças significativas entre as categorias. Além disso, não foram encontradas diferenças significativas entre as categorias na velocidade correspondente ao LAN.

\section{DISCUSSÃO}

A intensidade do OBLA, representada como $\% \mathrm{FC}_{\text {máx }}$, foi $87,0,86,3$ e $91,2 \% \mathrm{FC}_{\text {máx }}$ para as categorias Sub-17, Sub-20 e Profissional, respectivamente. De acordo com o American College of Sports Medicine (ACSM) ${ }^{22}$, as intensidades encontradas em \% $\mathrm{FC}_{\text {máx }}$ para as categorias sub-17 e Sub-20 são consideradas altas, enquanto que a intensidade encontrada para os profissionais é considerada muito alta. Deve-se atentar para o fato de que esta classificação de intensidade mesmo sendo em valores relativizados pela $\mathrm{FC}_{\text {máx }}$ foram recomendados para pessoas saudáveis, podendo não ser a melhor classificação para atletas.

Casajús ${ }^{14}$ identificou intensidades de $91 \% \mathrm{FC}_{\text {máx }}$ no LAN em jogadores profissionais espanhóis, utilizando o OBLA para a determinação do mesmo. Este valor se aproximou do encontrado para a categoria profissional do presente estudo. No entanto, no estudo de Casajús $^{14}$, a $\mathrm{FC}_{\text {máx }}$ foi determinada em laboratório, fato que pode ter subestimado a $\mathrm{FC}_{\text {máx }}$ $\mathrm{e}$, conseqüentemente, superestimado o LAN destes atletas.

As intensidades do LAN identificadas no presente estudo foram maiores em comparação com o estudo de Chin et al. ${ }^{15}$ que avaliaram 24 jogadores profissionais chineses e encontraram valores de $89 \% \mathrm{FC}_{\max }, 80 \% \mathrm{VO}_{2 \max }$ e $159 \mathrm{bpm}$ para o OBLA, sendo a $\mathrm{FC}_{\text {máx }}$ destes jogadores determinadas em laboratório. A falta de motivação dos atletas durante testes em laboratório

Tabela 1. Idade, estatura, percentual de gordura $(\% \mathrm{G})$ e capacidade aeróbia $\left(\mathrm{VO}_{2 \operatorname{máx}}\right)$ dos voluntários. Valores apresentados como média e desvio padrão.

\begin{tabular}{lccccc}
\hline Categoria & $\mathrm{n}$ & $\begin{array}{c}\text { Idade } \\
\text { (anos) }\end{array}$ & $\begin{array}{c}\text { Estatura } \\
(\mathrm{cm})\end{array}$ & $\% \mathrm{G}$ & $\begin{array}{c}\mathrm{VO} \mathrm{m}_{\mathrm{max}} \\
\left(\mathrm{mL} \cdot \mathrm{kg}^{-1} \cdot \mathrm{min}^{-1}\right)\end{array}$ \\
\hline Sub-17 & 19 & $16,4 \pm 0,5$ & $175 \pm 6,8$ & $9,3 \pm 1,0$ & $56,1 \pm 2,0$ \\
Sub-20 & 12 & $18,2 \pm 0,7$ & $178 \pm 7,3$ & $8,5 \pm 1,0$ & $50,2 \pm 2,9$ \\
Profissional & 14 & $24,3 \pm 3,9$ & $181 \pm 7,0$ & $9,1 \pm 1,3$ & $66,0 \pm 4,5$ \\
\hline
\end{tabular}

Tabela 2. $\mathrm{FC}_{\text {máx' }} \mathrm{FC}$, \%FC $\mathrm{Fáx}_{\text {ma }}$ e velocidade do limiar anaeróbio dos jogadores. Valores apresentados como média e desvio padrão.

\begin{tabular}{lccccccccc}
\hline Categorias & \multicolumn{3}{c}{ Sub-17 } & \multicolumn{3}{c}{ Sub-20 } & \multicolumn{3}{c}{ Profissional } \\
\hline $\mathrm{FC}_{\text {máx }}(\mathrm{bppm})$ & $202^{*}$ & \pm & 7 & 197 & \pm & 9 & 193 & \pm \\
$\mathrm{FC}(\mathrm{bpm})$ & 176 & \pm & 12 & 171 & \pm & 11 & 176 & \pm \\
$\% \mathrm{FC}_{\text {max. }}$ & $87,0^{*}$ & \pm & 1,1 & $86,3 *$ & \pm & 0,8 & 91,2 & \pm \\
Velocidade $(\mathrm{km} / \mathrm{h})$ & 12,1 & \pm & 1,3 & 12,1 & \pm & 1,1 & 1,2 & 1,9 & \pm \\
\hline
\end{tabular}

* Diferença significativa $(p<0,05)$ em relação à categoria profissional. 
pode fazer com que estes não atinjam o maior esforço possível, levando a uma subestimação da $\mathrm{FC}_{\text {máx }}{ }^{23}$. Assim, a $\mathrm{FC}_{\text {máx }}$ atingida durante testes em laboratório poderia estar sendo subestimada em relação a medida em competição ou testes de campo ${ }^{24}$. Portanto, os valores identificados por Chin et a ${ }^{16}$ podem estar superestimando o LAN dos jogadores avaliados. Estes autores sugerem que um jogo de futebol competitivo requer dos jogadores uma atividade próxima do LAN durante um período prolongado do jogo. Além disso, Miles et al..$^{12}$ relatam que, constantemente, a [Lactato] durante um jogo de futebol é superior a $4 \mathrm{mM}$ e consideram que um jogo de futebol representa um estímulo significativo para o aperfeiçoamento da capacidade aeróbia.

A intensidade correspondente ao OBLA do presente estudo foi similar aos valores encontrados por Vanfraechem e Thomas ${ }^{25}$. Estes autores avaliaram 18 jogadores profissionais de futebol em um teste de laboratório realizado em cicloergômetro, para a determinação de algumas variáveis fisiológicas indicativas do esforço. $\mathrm{O}$ limiar ventilatório ocorreu a $87 \% \mathrm{FC}_{\text {máx }}$, o que correspondeu a cerca de $80 \% \mathrm{VO}_{2 \text { máx }}$ e $146 \pm$ $9 \mathrm{bpm}$. Bunc et al. ${ }^{16}$ também determinaram o limiar ventilatório em jogadores da antiga Checoslováquia, durante testes de laboratório e encontraram valores correspondentes a 92,1 \pm $4,3 \% \mathrm{FC}_{\text {máx }}$. Leatt et al..$^{26}$ estudaram jogadores de futebol da mesma faixa etária dos jogadores sub17 e sub-20 que participaram do presente estudo e encontraram valores de LAN, obtidos através do limiar ventilatório em testes progressivos em laboratório, que corresponderam a $88 \% \mathrm{FC}_{\max }$. Estes valores também são similares aos valores encontrados no presente estudo. Mesmo considerando que os estudos citados tenham sido bem conduzidos e sejam relevantes para os profissionais que trabalham com treinamento físico no futebol, deve-se atentar para a dificuldade em se comparar os valores encontrados no presente estudo com o supra citado, dada a inespecificidade do teste em cicloergômetro em relação à modalidade esportiva avaliada.

Estudos têm encontrado diferenças entre valores de FC obtidos durante testes realizados em campo, em esteira e em cicloergômetro ${ }^{23,24}$. Além disso, as variações apresentadas entre os resultados dos estudos podem ser explicadas pela diferença entre as formas de identificação do LAN (lactacidemia versus ventilação). A explicação para o aumento exponencial na ventilação (limiar ventilatório) a partir de uma determinada intensidade de esforço é decorrente do aumento na concentração de gás carbônico sangüíneo derivado do tamponamento plasmático do ácido lático. Desta forma, o limiar ventilatório ocorre uma vez que a [Lactato] sangüínea já esteja elevada e portanto, teoricamente, deveria superestimar o LAN medido através da lactacidemia. No entanto, alguns estudos não têm encontrado diferenças significativas entre o limiar ventilatório e o limiar de lactato ${ }^{27,28}$ e entre o limiar ventilatório e o limiar de variabilidade da FC ${ }^{29}$, o qual também tem sido utilizado para estimar o LAN. Contudo, nenhum destes estudos comparou o limiar ventilatório com a MFEL que é considerada o padrão ouro para a identificação do LAN. O estudo de Van Schuylenbergh et al. ${ }^{8}$ foi o único encontrado que fez esta comparação e não foi encontrada diferença significativa entre as duas variáveis. No entanto, não houve correlação significativa entre a potência (Watts) correspondente à MFEL e ao limiar ventilatório. Além disso, a lactacidemia na MFEL foi 5,4mM e no limiar ventilatório $3,3 \mathrm{mM}$.

Santos ${ }^{13}$ encontrou uma velocidade média de limiar de $14 \mathrm{~km} / \mathrm{h}$ em jogadores da primeira divisão do futebol português. No presente estudo, os jogadores da categoria profissional obtiveram um valor médio de $12,9 \mathrm{~km} / \mathrm{h}$. Apesar da velocidade encontrada no presente estudo ter sido menor, deve-se ressaltar que os jogadores foram avaliados no período de pré-temporada, voltando de férias, quando ainda não estavam em seu melhor nível de condicionamento aeróbico. Balikian et al. ${ }^{17}$ também avaliaram a velocidade de corrida correspondente ao LAN em jogadores profissionais, comparando diferentes posições desempenhadas pelos jogadores em campo, determinaram que esta velocidade variou entre 12 e $14 \mathrm{~km} / \mathrm{h}$ para goleiros e jogadores de meio campo, respectivamente. No entanto, os jogadores avaliados pelos autores foram jogadores da segunda divisão do futebol brasileiro.

A discussão feita anteriormente sobre a relativização do esforço considerado como $\% \mathrm{FC}_{\text {máx }}$, deve ser observada quando os valores de LAN forem apresentados como \%FC máx $_{\text {. A }}$ análise de valores de FC absoluta não é a mais adequada para a comparação e avaliação da intensidade de esforço alcançada em uma situação entre indivíduos, para tal, valores percentuais 
devem ser adotados ${ }^{21}$. Portanto, caso a FC máx considerada para a relativização da FC do LAN não seja identificada durante testes adequados ou situações de esforço máximo real (competiÇão), os indicadores de LAN representados pelo $\% \mathrm{FC}_{\text {máx }}$ podem ser superestimados.

No presente estudo, os valores absolutos de FC no LAN não foram diferentes nas três categorias. No entanto, quando a análise foi feita através do \%FC ${ }_{\text {máx }}$ os valores do LAN encontrados para a categoria profissional foram maiores que nas demais categorias. Este fato reforça a importância de se analisar o LAN como \%FC ${ }_{\text {máx }}$ e não como valores absolutos.

A FC ${ }_{\text {máx }}$ da categoria profissional foi menor que a da categoria juvenil. Este fato pode ser explicado pela diminuição da $\mathrm{FC}_{\text {máx }}$ com o aumento da idade ${ }^{23}$. Sugere-se, também, que possa ocorrer uma diminuição da $\mathrm{FC}_{\text {máx }}$ de atletas decorrente de adaptações ao treinamento aeróbico $^{30}$, no entanto, tal interpretação pode ser dependente do método utilizado para avaliar este parâmetro de rendimento em testes fisiológicos de maneira relativizada ou absoluta.

Levando em conta as considerações acima mencionadas, reforça-se a idéia de que durante a prescrição das cargas de treinamento, devese priorizar a individualização das mesmas. Este fato pode ser confirmado pela ausência de diferença significativa entre o LAN quando foi apresentado em valores absolutos de FC, sendo que o mesmo não ocorreu quando essa comparação foi feita em valores de $\% \mathrm{FC}_{\mathrm{Max}}$.

Além disso, sabendo que o LAN é um fator fisiológico utilizado para avaliação e seleção de atletas, a fase da temporada em que os testes se encontram pode ser determinante para uma conclusão mais fidedigna sobre a aptidão dos avaliados, tendo em vista que o LAN avaliado como velocidade dos jogadores foi menor em comparação com outros estudos, uma vez que os atletas deste estudo se encontravam em fase inicial de treinamento de uma temporada de competição, o que não aconteceu quando os valores de LAN dos atletas deste estudo foram expressos como \%FC ${ }_{\text {Max }}$ ou FC absoluta.

\section{CONCLUSÃO}

Conclui-se com o presente estudo que o LAN de jogadores de futebol é, em média, $87 \%$ FCmáx, que é uma intensidade considerada alta. A intensidade correspondente ao OBLA apre- sentada como \% $\mathrm{FC}_{\text {máx }}$ é maior em jogadores da categoria profissional, quando comparada aos jogadores das categorias sub-17 e sub-20. Entretanto, quando esta mesma comparação utiliza valore absolutos de FC esta diferença não é encontrada. A velocidade correspondente ao LAN do presente estudo é baixa em relação a encontrada em outros estudos. Esta diferença, provavelmente, se deve aos testes terem sido empregados no inicio da pré-temporada.

\section{REFERÊNCIAS BIBLIOGRÁFICAS}

1. Bangsbo J. The physiology of soccer, with special reference to intense intermittent exercise. Acta Physiol Scan 1994;151: suppl 619.

2. Wisloff U, Helgerud J, Hoff J. Strength and Endurance of elite soccer players. Med Sci Sports Exerc 1998;30(3): 462-467.

3. Ekblom B. Applied Physiology of Soccer. Sports Med 1986;3:50-60.

4. Helgerud J, Engen LC, Wisloff U, Hoff J. Aerobic endurance training improves soccer performance. Med Sci Sports Exerc 2001;33(11):1925-1931.

5. Casajús AJ. Seasonal variation in fitness variables in professional soccer players. J Sports Med Phys Fitness 2001;41:463-469.

6. Pate RR, Kriska A. Physiological basis of the sex difference in cardiorespiratory endurance. Sports Med 1984;1:87-98.

7. Bosquet L, Léger L, Legros P. Methods to determine aerobic endurance. Sports Med 2002; 32(11):675-700.

8. Van Schuylenbergh R, Vanden Eynde B, Hespel P. Correlations between lactate and ventilatory thresholds and the maximal lactate steady state in elite cyclists. Int J Sports Med 2004;25(6):403-408.

9. Sjodin B, Jacobis I. Onset of blood lactate accumulation and marathon running performance. Int J Sports Med 1981;2(1):23-26.

10. Heck H, Mader A, Hess G, Mucke S, Muller R, Hollmann W. Justification of the 4-mmol/L lactate threshold. Int J Sports Med 1985;61:219-224.

11. Denadai BS, Gomide EB, Greco CC. The relationship between onset of blood lactate accumulation, critical velocity, and maximal lactate steady state in soccer players. J Strength Cond Res 2005;19(2):364-368.

12. Miles A, Maclaren D, Reilly T. Yamanaka, K. An analysis of physiological strain in four-a-side women's soccer. J Sports Sci 1992;10:142-143.

13. Santos JAR. Estudo comparativo, fisiológico, antropométrico e motor entre futebolistas de diferente nível competitivo. Rev Paul Educ Fis 1999;13(2):146-150

14. Casajús A J. Seasonal variation in fitness variables in professional soccer players. J Sports Med Phys Fitness 2001;41:463-469. 
15. Chin M-KLO, Y SA, Li CT, So CH. Physiological profiles of Hong Kong elite soccer players. Br J Sports Med 1992;26(4):262-266.

16. Bunc V, Heller J, Leso J, Sprynarova S, Zdanowicz R. Ventilatory threshold in various groups of highly trained athletes. Int J Sports Med 1987;8:275-280.

17. Balikian P, Lourenção A, Ribeiro LFP. Festuccia WTL, Neiva CM. Consumo máximo de oxigênio e limiar anaeróbio de jogadores de futebol: comparação entre as diferentes posições. Rev Bras Med Esporte 2002;8:32-36.

18. Mcardle WP, Katch FI, Katch VL. Fisiologia do Exercício, Energia, Nutrição e Desempenho Humano.4 ed. Williams \& Wilkins; 2003.

19. Margaria R, Aghemo P, Pinera LF. A simple relation between performance in running and maximal aerobic power. J Appl Physiol 1975;38(2):351-352.

20. Eniseler, N. Heart Rate and Blood Lactate Concentrations as Predictors of Physiological Load on Elite Soccer Players During Various Soccer Training Activities. J Strength Cond Res 2005;19(4):799-804.

21. Karvonen J, Vuorimaa T. Heart rate and exercise intensity during sports activities: practical application. Sports Med 1988;5:303-312.

22. ACSM - American College of Sports Medicine. Position Stand. The Recommended quality of exercise for developing and maintaining cardiorespiratory and muscular fitness, and flexibility in healthy adults. Med Sci Sports Exerc 1998;30(6):975-991.

23. Antonacci L, Mortimer LACF, Rodrigues V, Coelho DB, Soares DD, Silami-Garcia E. Competition, estimated, and test maximum heart rate. J Sports Med Phys Fitness 2007;47: 418-421.

24. Boudet G, Garet M, Bedu M, Albuisson E, Chamoux A. Median maximal heart rate for calibration in different conditions: Laboratory, Field and Competition. Int J Sports Med 2002; 23(4):290-297.
25. Vanfraechem JHP, Thomas M. Maximal aerobic power and ventilatory threshold of a top level soccer team of a top-level soccer team. J Sports Sci 1992;10:149-156.

26. Leatt P, Shephard RJ,; Plyley MJ. Specific Muscular development in under -18 soccer players. J Sports Sci 1987;5:165-175.

27. Wyatt F, Godoy S, Autrey L, McCarthy J, Heimdal J. Using a logarithmic regression to identify the heart-rate threshold in cyclists. J Strength Cond Res 2005;19(4):838-841.

28. Belli T, Ackermann MA, Ribeiro LF, Langeani R, Galdino da Silva R, Baldissera V. Lactate and ventilatory thresholds in type 2 diabetic women. Diabetes Res Clin Pract 2007;76(1):18-23.

29. Cottin F, Médigue C, Lopes P, Leprêtre PM, Heubert R, Billat V. Ventilatory thresholds assessment from heart rate variability during an incremental exhaustive running test. Int J Sports Med 2007;28(4):287-294.

30. Zavorsky GS. Evidence and Possible Mechanisms of Altered Maximum Heart Rate With Endurance Training and Tapering. Sports Med 2000;29(1):13-26.

\section{Agradecimentos}

Apoio financeiro: CNPq, CAPES, FAPEMIG.

\author{
Endereço para correspondência \\ Prof. Daniel Barbosa Coelho \\ Rua Prof. Antonio Aleixo, 500/2002, Lourdes \\ 30180-150 - Belo Horizonte, MG \\ E-mail: danielcoelhoc@bol.com.br
}

